\title{
Comment vivre ensemble de Roland Barthes
}

Vie et mort d'un site littéraire

\section{Claude Coste}

\section{OpenEdition}

\section{Journals}

Édition électronique

URL : https://journals.openedition.org/recherchestravaux/107

DOI : 10.4000/recherchestravaux.107

ISSN : 1969-6434

Éditeur

UGA Éditions/Université Grenoble Alpes

Édition imprimée

Date de publication : 15 juin 2008

Pagination : 201-215

ISBN : 978-2-84310-125-0

ISSN : 0151-1874

\section{Référence électronique}

Claude Coste, «Comment vivre ensemble de Roland Barthes », Recherches \& Travaux [En ligne], 72 |

2008, mis en ligne le 15 décembre 2009, consulté le 21 septembre 2021. URL : http://

journals.openedition.org/recherchestravaux/107 ; DOI : https://doi.org/10.4000/recherchestravaux. 107 


\section{Comment vivre ensemble de Roland Barthes : vie et mort d'un site littéraire}

Le premier cours de Barthes au Collège de France, donné de janvier à mai I 977, porte un titre et un sous-titre bien étranges : Comment vivre ensemble: simulations romanesques de quelques espaces quotidiens. Nouvellement élu dans une des plus prestigieuses institutions françaises, Barthes choisit un sujet (la sociabilité) et une méthode (le romanesque) qui témoignent de son indépendance critique, loin de tout dogmatisme et de toute provocation. Je me propose dans cet article de suivre un double objectif. Le premier consiste en la rapide présentation d'un cours atypique, autant par le contenu que par la démarche intellectuelle; le second m'amènera à décrire et à analyser le processus éditorial qui, à partir des manuscrits conservés à l'IMEC (Institut Mémoires de l'édition contemporaine), a conduit à la publication d'un livre (Seuil, 2002) ${ }^{\mathrm{I}}$, puis à la réalisation d'un site Internet, www.roland-barthes.com (Seuil, 2003)', qui est resté en ligne jusqu'à l'automne 2006 et dont il faut désormais parler au passé. Je reviendrai à l'issue de ce parcours sur les raisons d'un tel échec.

Le dossier du Comment viure ensemble comporte un ensemble composé de trois types de documents : les notes de cours manuscrites (une centaine de

I. R. Barthes, Comment vivre ensemble : simulations romanesques de quelques espaces quotidiens, édité par Cl. Coste, sous la direction d'É. Marty, Seuil/IMEC, «Traces écrites», 2002. Parallèlement aux livres, le Seuil publie, en 2002, sous forme discographique, l'enregistrement des trois cours au Collège de France Comment vivre ensemble, Le Neutre, La Préparation du roman.

2. La conception de ce site a reposé sur la collaboration des éditions du Seuil (M.-F. Fontaine, V. Grancher) et de la société informatique QWAM. J'ai assuré la responsabilité du travail éditorial, secondé par une équipe d'étudiants des Universités de Caen, Paris VII et Amiens (R. Boulaâbi, A. Garnier, B. Leprêtre, M. Meyre, Y. Thommerel). 
pages), l'enregistrement des quatorze séances, d'une heure chacune, effectué par les étudiants (manque la dernière demi-heure), une cinquantaine de fiches préparatoires accompagnées d'une bibliographie. S'ajoutent à cela les notes destinées au séminaire que Barthes a donné parallèlement à son cours et qui n'a pas été enregistré. Intitulée Tenir un discours, cette vingtaine de pages développe une réflexion générale sur les intimidations de langage, puis analyse quelques exemples empruntés à la littérature (tirade d'Andromaque à Hermione dans l'Andromaque de Racine, tirade de Charlus au narrateur dans Le Côté de Guermantes).

Quel sort pouvait-on réserver à cet ensemble exceptionnel ouvert aux chercheurs, mais difficilement consultable par un large public d'amateurs ou d'étudiants? Il aura fallu presque vingt-cinq ans pour que ce travail de Barthes, conçu pour une diffusion orale et pour un public restreint, trouve une nouvelle vie grâce à deux formes de diffusion, le livre et le site. Ces deux modes d'expression, à la fois différents et complémentaires, étaient conçus pour se combiner, apportant la preuve que chaque support correspond à un usage particulier et que le livre a encore de beaux jours à côté de l'informatique. Cette collaboration des différents modes de diffusion, naturellement, ne prend son sens que par rapport aux documents manuscrits et sonores : la conception de chaque support, la confrontation de l'un et de l'autre, comme le passage de l'un à l'autre, tentaient de répondre au défi éditorial lancé par le travail de Barthes et l'état de ses archives. Depuis Le Degré zéro de l'écriture et les pages célèbres consacrées à la responsabilité de la forme, on sait combien Barthes se refuse à séparer le contenant et le contenu, le message et son support, l'oral et l'écrit. Une telle éthique de l'écriture, qu'il s'agisse de littérature ou de critique, s'impose et en impose à l'éditeur d'un document aussi complexe, sur le plan matériel et intellectuel, que les cours de Barthes au Collège de France. Autrement dit, à tel objet, telle édition - ou telles éditions.

\section{Idiorrythmie}

Dans sa Leçon inaugurale, Barthes revendique le droit de fonder la recherche sur un fantasme. Un peu plus tard, dans La Chambre claire, il affirmera tout aussi nettement : «J'ai toujours eu envie d'argumenter mes humeurs.» En assumant pleinement sa subjectivité, le chercheur ne dérive pas du côté de l'égotisme ou de la confession impudique : il rappelle utilement que la valeur fonde le savoir et que le savoir sauve la valeur en lui donnant une forme communicable. En d'autres termes, si l'affect lance la recherche, il trouve en elle la sécurité du code et le réconfort de la durée. 
Le fantasme à l'origine du Comment vivire ensemble s'incarne dans un mot, un mot un peu étrange que Barthes rencontre en lisant L'Été grec de Jacques Lacarrière. Le désir récurrent d'une sociabilité singulière, obsession jusque-là flottante et instable parce que sans signifiant pour lui donner forme, se condense soudain, par le hasard des lectures, dans le mot «idiorrythmie». Appartenant au vocabulaire religieux, ce mot d'«idiorrythmie» désigne une organisation monacale très particulière, caractéristique du mont Athos. Il renvoie au rythme de vie de certains moines, rattachés à un monastère, mais vivant le plus souvent seuls, en marge de la communauté. Cette institution, qui se situe à mi-chemin entre érémitisme et cénobitisme, combine l'indépendance de l'individu et l'appartenance au groupe. Au-delà de sa signification religieuse, le mot d'idiorrythmie séduit Barthes par sa capacité à donner une forme verbale au fantasme de sociabilité qui l'habite. Grâce aux vertus de la métaphore, le mot sert de fil conducteur à l'exploitation systématique d'un désir : le rêve d'une vie à la fois solitaire et collective, d'un timing heureux où s'harmonisent le rythme de l'individu et celui de la communauté.

Porté par son projet, Barthes imagine donc devant les auditeurs du Collège de France une vaste maison située au bord de la mer, dans un paysage méditerranéen, qu'on louerait entre amis et dans laquelle chacun disposerait d'une chambre particulière. Mais la confidence personnelle tourne rapidement court : pour donner forme et durée à cet idéal de vie, Barthes préfère passer par la littérature, qu'il utilise comme un vaste répertoire d'expérimentations fictives. S'il n'existe pas vraiment de textes consacrés à l'idiorrythmie - que l'on prenne le mot au sens propre ou métaphorique -, Barthes réussit à constituer un corpus très hétérogène où cette notion apparaît, ne serait-ce que par éclats ou par bouffées. La fin de la «Présentation» est consacrée à une rapide description des textes sur lesquels le professeur fondera la dynamique de son cours. On trouve ainsi des ouvrages religieux portant sur la vie monacale dans le christianisme ou à Ceylan, des analyses sociologiques, et surtout un ensemble de textes littéraires, généralement narratifs, choisis en fonction d'un lieu caractéristique : La Montagne magique de Thomas Mann pour le sanatorium, Robinson Crusoé de Daniel Defoe pour l'île, Pot-Bouille d'Émile Zola pour l'immeuble bourgeois, La Séquestrée de Poitiers d'André Gide pour la chambre.

À partir de ce corpus, le travail de recherche se construit librement, organisé en «traits» comme Fragments d'un discours amoureux l'était en «figures». De séance en séance, Barthes enchaîne - ou plutôt juxtapose - des développements plus ou moins longs, classés par ordre alphabétique : "Acédie», «Athos», «Couple», «Saleté»... Le cheminement s'achève quatorze séances plus tard sous la forme d'une conclusion assez désenchantée. Le comment 
vivre ensemble idiorrythmique est-il possible? Ne relève-t-il pas tout simplement de l'utopie? La réponse est doublement ambiguë : comme utopie, c'està-dire comme échec de l'idéal, le fantasme porte en lui une dynamique qui stimule la création et la pensée. Il est pour Barthes un usage stratégique de l'utopie, caractérisé moins par la déception que par la stimulation. Mais peuton véritablement parler d'une utopie du vivre ensemble? Il n'est pas certain que ce fantasme accède vraiment à ce statut ambivalent mais rassurant. En effet, comme le fait remarquer Barthes, il n'est d'utopie que de la vie collective. Fantasme de l'individu solitaire et solidaire, l'idiorrythmie ne peut en aucune manière se comparer aux vastes constructions fouriéristes ou sadiennes. Pour le meilleur et pour le pire, la singularité exclut le système, la précarité accompagne la liberté. Toute la difficulté de l'entreprise idiorrythmique est de trouver le bon Télos, la bonne «Cause» (comme le traduit Barthes lui-même), c'est-àdire la bonne raison pour rassembler un groupe d'individus. Comment trouver un projet suffisamment fort pour rapprocher les gens sans s'aliéner à un discours contraignant, qu'il soit politique, religieux ou idéologique? Autrement dit, l'entreprise idiorrythmique peut-elle trouver en elle-même sa propre justification? Le cours ne répond pas clairement, mais le doute s'installe peu à peu... Minée par la crainte ou par l'échec, la quête de Barthes laisse apparaittre un autre fantasme qui est comme l'ombre portée ou le double du premier. À l'idiorrythmie se combine un autre désir beaucoup moins ouvert sur le monde : un désir obsessionnel de réclusion, de repli protecteur, qui trouve dans La Séquestrée de Poitiers son expression la plus fascinante. Quand le monde devient menace, quand la délicate harmonie de l'idiorrythmie révèle ses limites tant matérielles qu'intellectuelles, il ne reste plus qu'à s'enfouir sous les couvertures, se replier dans sa coquille, se laisser emporter par le vertige d'une réclusion salutaire.

Il existe pourtant une exception : la relation enseignante elle-même. N'estce pas sur ce plan-là - et uniquement sur ce plan là - que l'on peut envisager un dialogue harmonieux entre l'individu et le groupe, le professeur et l'auditoire du Collège de France? Barthes aimait les cours magistraux, préférant la tirade de l'enseignant à l'échange pédagogique direct, qu'il ne se sentait pas capable de conduire ou, plutôt, dont il mesurait la vanité. Mais en occupant la chaire et en monopolisant la parole, le professeur n'exclut pas les autres, bien au contraire, puisque c'est pour eux et par eux que le cours existe. Conçu dans la solitude du bureau, diffusé sous la forme d'un immense discours prolongé de semaine en semaine, le cours selon Barthes intègre la communauté comme idée conductrice, fait du destinataire cet objet de pensée qui donne forme au message et conduit le cheminement intellectuel. C'est finalement l'espace même du cours qui, dans un domaine très limité, réalise le projet d'idiorrythmie, la conjugaison 
d'une parole solitaire et d'une écoute collective. On se souvient d'une phrase un peu ironique de l'amoureux des Fragments: «Je parle, tu m'écoutes, donc nous sommes.»

Comme seul accomplissement réussi de l'idiorrythmie, la relation enseignante met l'accent sur la littérature et la pensée comme lieu et forme du partage, comme manifestation intellectuelle de ce repli sur soi que la couverture et la chambre symbolisaient si bien dans le cours. Quand la Séquestrée de Poitiers nie le monde et se recompose un monde dans la clôture de sa chambre, la communication verbale crée un échange singulier qui se tient à distance de la réalité tout en entretenant une étroite relation avec elle. En instaurant une communication différée, la littérature, écrite, prolonge encore ce mouvement d'éloignement à l'égard des autres - mais sans rompre le dialogue.

Dans ce dialogue idiorrythmique, le roman joue un rôle essentiel : romans du corpus, roman fantasmé dont Barthes étudiera bientôt la «Préparation». Mais si le roman ne surprend pas comme objet d'investigation, qu'en est-il de sa relation avec la méthode, qu'y a-t-il de romanesque dans ce cours? Comment associer deux entreprises que notre culture universitaire place aux antipodes : la recherche intellectuelle et la fiction narrative? On trouvera une première réponse du côté de Paul Ricœur. Des pages célèbres de Temps et récit, en effet, opposent deux conceptions du temps totalement différentes : une conception objective et collective, représentée par Aristote, et une conception subjective et individuelle, telle que la définit saint Augustin. Selon Ricœur, le récit, dans le monde occidental, se donne pour principale fonction d'accorder ces deux temporalités souvent antagonistes, d'articuler l'un avec l'autre le temps singulier du héros et le temps collectif de la société. Une belle scène du Lucien Leuwen de Stendhal illustre parfaitement le désir et la difficulté de concilier ce qui est presque inconciliable : défilant avec son régiment sous les fenêtres de la mystérieuse madame de Chasteller, le héros s'immobilise fasciné, oublie ses camarades et la laideur du monde; quand la colonne des cavaliers repart à son insu, il est brutalement arraché à sa contemplation et jeté au sol par son cheval. Aux prises avec une double temporalité, Lucien Leuwen a bien du mal à accorder le rythme personnel de son désir et le rythme collectif d'un régiment sans pitié pour sa singularité. On peut le dire autrement: Lucien Leuwen, comme tant de personnages romanesques, éprouve les plus grandes difficultés du monde à défendre cette «idiorrythmie» dont Barthes fera le terme conducteur de son cours. Fondé sur un fantasme du professeur, le Comment vivre ensemble entre ainsi en résonance avec une des grandes fonctions du récit. Du roman au cours, de la quête conciliatrice du personnage à la solitude librement interrompue du sujet barthésien, le glissement n'est ni artificiel, ni paradoxal : en se lançant dans une longue simulation romanesque de son 
fantasme, la démarche intellectuelle du cours entre en résonance avec une problématique essentielle de l'histoire du roman.

Mais Barthes n'est pas avare de paradoxes. Proche sans le dire de Ricœur, il manifeste son autonomie en valorisant le romanesque au détriment du roman, en proposant une temporalité, un timing, ou un muthos, en miettes. Sous-titré "Simulations romanesques de quelques espaces quotidiens», le Comment vivre ensemble expérimente à l'oral un romanesque en marge du roman, c'est-à-dire un roman sans récit. Dans son cours, Barthes ne raconte pas une histoire. La succession des «traits» le dit bien : l'organisation obéit à une structure thématique ("Akèdia», "Anachôrèsis», "Animaux», "Athos», «Autarcie»...) et non pas narrative, toute forme de métonymie se voyant congédiée par l'arbitraire de l'ordre alphabétique³. Que reste-t-il donc quand on écrit ou que l'on parle un roman sans récit? De quoi le romanesque du cours peut-il bien être fait? Le mot «simulations» nous engage du côté de la «maquette», c'est-à-dire de cette construction imaginaire et bien réelle à la fois qu'un romancier dessine avant de commencer à écrire. Lieu habitable par la fiction, la «maquette», selon Barthes, est le décor romanesque qui précède le roman (le désert, la chambre, l'immeuble...). Dans ces «maquettes» transformées en décors intellectuels, les objets et les personnages investissent les lieux et les ouvrent à une temporalité segmentée. Comme l'écrit Barthes dans le trait «Événement», Robinson Crusoé perd de son intérêt avec l'arrivée des sauvages, avec le retour en Europe, c'est-à-dire avec le développement du roman d'aventures. L'événement a sa place dans la «maquette», mais au singulier ou dans le désordre de la fragmentation : événements ténus, désaccordés, insignifiants, évitant à la fois le devenir narratif et le sens justificatif. Comme tout romancier, Barthes montre plus qu'il ne démontre, court-circuite la pensée avant qu'elle ne devienne trop abstraite, avant que la «maquette» ne devienne symbole. Le romanesque du Vivre-ensemble nait dans des lieux encombrés d'objets (fleurs, déchets, tables, chaises, bures...) où se donnent à voir les gestes ordinaires de personnages souvent extraordinaires (un stylite, une séquestrée, un naufragé...).

3. R. Barthes s'explique clairement sur ce choix stratégique dans l'avant-propos de Fragments d'un discours amoureux : «On a donc soumis la suite des figures (inévitable puisque le livre est astreint, par statut, au cheminement) à deux arbitraires conjugués : celui de la nomination et celui de l'alphabet. Chacun de ces deux arbitraires est cependant tempéré : l'un par la raison sémantique (parmi tous les noms du dictionnaire, une figure ne peut en recevoir que deux ou trois), l'autre par la convention millénaire qui règle l'ordre de notre alphabet. On a évité ainsi les ruses du hasard pur, qui aurait bien pu produire des séquences logiques; car il ne faut pas, dit un mathématicien, "sous-estimer la puissance du hasard à engendrer des monstres"; le monstre, en l'occurrence, eût été, sortant d'un certain ordre des figures, une "philosophie de l'amour", là où il ne faut attendre que son affirmation.» (Seuil, «Tel Quel», I 977, p. I I-I 2) 
«J'ouvre seulement un dossier» : par cette formule lancinante, Barthes assume pleinement le projet intellectuel de son cours. Comme le romancier, le professeur dégage une forme, sans aller jusqu'au bout de son interprétation, lance des idées, ouvre des pistes, amorce un développement que l'auditeur est appelé à poursuivre lui-même à partir des matériaux donnés par le cours. Une telle démarche lance un appel à l'auditeur et un défi à l'éditeur.

\section{Vers le livre}

La publication du Comment vivre ensemble s'inscrit dans un vaste projet d'édition qui, sous la direction d'Éric Marty, couvre les trois cours au Collège de France ${ }^{4}$, le séminaire sur $S / Z$, le séminaire sur le «Fichier d'auteur» (qui deviendra Roland Barthes par Roland Barthes) et le séminaire sur le «Discours amoureux'». Cette entreprise collective met fin à plus de vingt ans d'attente. La médiocrité de la transcription d'un passage tiré du Neutre publiée de façon illégale par Bernard-Henri Lévy dans La Règle du jeu appelait une véritable réflexion sur le support adéquat. Comment faire pour diffuser un document manuscrit et sonore qui n'a aucun équivalent dans toute l'œuvre de Barthes? Comment faire pour tenir compte de la distinction fondamentale qu'opère Barthes entre l'oral et l'écrit, l'écriture pleinement élaborée et la simple transcription de la parole du professeur (ce qu'il appelle la «scription»)?

La solution d'une édition multimédia s'est peu à peu imposée comme la plus respectueuse des divers modes d'expression, oraux (l'enregistrement des séances) et écrits (les notes de cours manuscrites). L'ITEM et l'IMEC se lancèrent dans la conception d'un prototype. À partir de la première séance de cours, un magnifique document, sonore et visuel ${ }^{7}$, a montré combien ce support convenait à un mode d'expression comme le cours et à un penseur comme Barthes. Conçu comme un essai, ce produit qui n'était pas destiné à la commercialisation n'a pas connu de suite. Que restait-il à faire? La solution

\footnotetext{
4. Voir note I.

5. Tous ces séminaires ont été dispensés à l'École pratique des hautes études. Est paru Le Discours amoureux, séminaire à l'École pratique des hautes études, 1974-1976, suivi de Fragments d'un discours amoureux : inédits, avant-propos d'É. Marty, présentation et édition de Cl. Coste, Seuil, coll. «Traces écrites», 2007.

6. Il s'agissait d'une transcription de la version orale. L. Dispot, «Roland Barthes : Le Désir de neutre», La Règle du Jeu, n' 5, août I 99 I, p. 36-60.

7. Ce prototype a été conçu par É. Marty et N. Léger avec la collaboration de l'informaticien A. Giffard. Il s'agissait de la reproduction en fac-similé de quelques pages manuscrites de Comment vivre ensemble, accompagnée par un apparat critique et de l'enregistrement correspondant.
} 
du fac-similé écartée - très coûteuse et peu lisible -, les responsables optèrent pour une édition imprimée des notes de cours et une édition discographique des enregistrements. Grâce à la compression des fichiers que permet la technique $\mathrm{MP}_{3}$, il a été possible de faire tenir sur un seul $\mathrm{CD}$ la totalité des quatorze heures de cours.

En acceptant que son cours soit enregistré, Barthes acceptait de fait que son travail, sa voix, la lettre de sa parole, continuent à vivre au-delà du présent de la séance. L'édition discographique s'imposait donc; mais, quoique fidèle au message originel et original, cette solution proposait un produit peu pratique d'usage, ne permettant pas une circulation facile d'un passage à l'autre. De plus, une diffusion exclusivement discographique aurait exclu les séminaires dont nous ne conservons que les notes manuscrites. Si le cours de Barthes devait devenir un objet de travail pour les spécialistes, les étudiants et les lecteurs désintéressés, il fallait donner au public un document facile à manier comme peut l'être un volume imprimé. Mais il n'était pas question de rédiger à la place de Barthes, de se substituer à son écriture, d'achever l'inachevé. Inversement, ces notes de cours ne pouvaient être livrées telles quelles, sans le secours d'un travail de transposition et d'un apparat critique conséquent. Entre littéralité et reformulation, la marge de manœuvre était assez étroite.

Les manuscrits, écrits très lisiblement à l'encre bleue, ne présentent aucune difficulté de déchiffrement. La compréhension littérale du texte demande par contre davantage d'efforts. Barthes ne rédigeait pas ses cours, et les notes, conçues pour le professeur, ne peuvent en aucune manière se comparer à un texte écrit pour la publication. Le travail de Barthes ne se présente pas non plus comme un canevas pédagogique, un aide-mémoire destiné à conduire l'improvisation. Les notes de cours offrent des états de rédaction très divers. À des passages syntaxiquement construits, à des paragraphes entièrement rédigés, succèdent de pures énumérations, de simples séries de mots. Mais si le manuscrit multiplie les ellipses (du verbe et des liens logiques), appelle une accommodation permanente de l'intelligence et de l'œil, la compréhension ne fait jamais défaut.

Les notes de cours, désormais placées au centre de l'entreprise éditoriale, appelaient un minutieux travail de transcription. Barthes «idolâtrait» la phrase : on a donc transcrit les notes en se donnant sans cesse la phrase comme unité formelle. Chaque fois qu'il a été possible de faire tenir une structure syntaxique idiomatique et clairement identifiable entre le double butoir de la majuscule et du point, l'entreprise a été tentée. L'aspect visuel du texte en a forcément été modifié, la ponctuation a été entièrement révisée. Mais à aucun moment, le choix de l'éditeur n'est venu forcer l'ambiguïté de la notation. 
Toutes les fois qu'il aurait fallu interpréter, le texte a été reproduit tel quel. Dans ses notes, plus encore que dans ses publications, Barthes multiplie les «: » et le «; ». Le manuscrit enchaîne souvent une cascade de remarques dont l'articulation logique n'est exprimée que par le deux-points. Il n'était guère possible de laisser en l'état ce trait d'écriture, qu'on trouvait certes déjà dans Roland Barthes par Roland Barthes, mais qui prend dans le cours une très grande ampleur. Le goût de Barthes pour les majuscules, les guillemets et les italiques (les soulignements dans le manuscrit), est tout aussi avéré. Devant leur démultiplication dans les notes, deux attitudes étaient possibles : reproduire les signes tels quels au risque de brouiller la lecture ou prendre le parti de les supprimer tous. On a choisi la seconde solution par un double souci de lisibilité, pour le lecteur, et de respect, pour l'auteur. Du manuscrit au texte écrit, de la profusion à l'économie, Barthes aurait opéré un tri que l'on n'a pas voulu effectuer à sa place. Les guillemets et les italiques n'ont été conservés que pour les mots dont la valeur conceptuelle, expressive, ou citationnelle, n'était guère contestable.

Cette ambition de lisibilité a commandé l'ensemble des modifications opérées sur le manuscrit : rédaction des mots (particulièrement des titres) abrégés dans le corps du texte ou dans les marginalia (Robinson Crusoé pour RC, À la Recherche du temps perdu pour RTP...), aération de la page, rationalisation de la numérotation. La translittération des mots grecs posait sans doute davantage de problèmes, puisqu'elle touche au signifiant, qu'elle fait fi de cette lenteur de déchiffrement que Barthes, dans sa «Présentation», revendique comme une attitude subversive dans un monde dominé par la vitesse. Malgré ces scrupules, la connaissance des langues anciennes a régressé de nos jours au point d'imposer la translittération comme la solution la moins mauvaise.

Sur la conception de l'apparat critique définie collectivement par l'équipe éditoriale, je m'explique longuement dans la préface du cours et dans un texte d'hommage à Barthes publié par le Collège de France ${ }^{8}$. Le Comment vivre ensemble offre par rapport aux autres cours une difficulté particulière qui tient au statut ambigu de l'érudition barthésienne. De façon générale, on ne trouve pas élucidées en note les références qu'on trouverait dans un dictionnaire courant des noms propres et des noms communs. Portant en grande partie sur la vie monastique au Moyen Âge, c'est-à-dire sur un domaine peu connu du grand public, le texte de Barthes appelait de nombreux éclaircissements que j'ai donnés en note. Mais inversement, le savoir érudit, pris dans une atmosphère à la fois respectueuse et ludique, ne donne jamais lieu chez Barthes à une exploitation systématique. Il aurait été un peu ridicule dans ce 
contexte-là de multiplier et de développer des notes dont la prolifération se serait inscrite à contre-courant d'une démarche d'amateur, parfaitement assumée par le professeur. C'est entre ces deux écueils, le trop et le pas assez, que j'ai dû naviguer en concevant l'apparat critique.

\section{Vers le site Internet}

L'édition papier n'est pas une fin en soi. Dans le prolongement de cette première entreprise, les éditions du Seuil ont souhaité réaliser un site Internet consacré aux trois cours au Collège de France (soit 55 heures d'enregistrement). À terme, si les premières expériences s'étaient révélées concluantes (avec d'abord Comment vivre ensemble, puis avec les deux autres cours), c'est toute l'œuvre de Barthes qui aurait été consultable en ligne. En tant qu'éditeur du premier cours, j’ai été étroitement associé à cette nouvelle entreprise, dont la responsabilité éditoriale m'a été confiée. Une maquette fonctionnelle a d'abord été réalisée, à partir des deux premières séances de cours et de la dernière. Puis, le travail a été étendu à la totalité du cours, qui a été mis en ligne avec l'URL suivante : www.roland-barthes.com.

Le site donnait accès (il faut désormais parler au passé!) à quatre types de documents : l'enregistrement des cours, leur transcription écrite (ce que Barthes appelle la «scription»), la reproduction des manuscrits en fac-similé, et leur transcription (il s'agit du texte publié en livre). La recherche multimédia autorise ainsi la mise en relation de documents très divers grâce à plusieurs modes d'expression (texte, son, image) tous liés à un même moment de l'enseignement. L'environnement de travail pour chacun des cours, dans le projet final comme dans la maquette, s'organisait selon trois grands types de fonctionnalités. Pour se guider dans sa lecture et dans son écoute, l'utilisateur pouvait visualiser le schéma de structure des documents, l'arborescence des éléments hiérarchisés du texte (titres de chapitres, sous-chapitres, etc.). Après ce premier repérage, l'utilisateur avait la possibilité de naviguer dans le cours de Barthes, en choisissant une version (fac-similé ou transcription du manuscrit, archives sonores $\left.{ }^{9}\right)$ ou en sélectionnant une «entrée» particulière : noms, mots clés (ou concepts), bibliographie, dates des cours, etc. Les deux termes de l'alternative (versions du cours ou «entrées») conservaient toute leur indépendance, aucune des deux ne prévalant sur l'autre. Il était possible de faire défiler sur l'écran la totalité du manuscrit page par page. Par contre, cette

9. Pour ne pas faire concurrence à l'édition papier et au CD-ROM, on ne pouvait pas accéder à la transcription des manuscrits et aux archives sonores dans leur continuité, mais seulement par fragments, par des requêtes ponctuelles sur des mots-clés. 
option n'existait pas pour la transcription des notes manuscrites ou la version orale, l'utilisateur étant appelé à se référer au livre publié ou aux enregistrements discographiques pour suivre ces versions en continu. Par sa capacité à totaliser et à mettre en relation les différents modes d'expression, le site fédérait sans les annuler les différentes formes de publication.

Après avoir, grâce à l'aide d'une équipe d'étudiants, pratiqué une transcription aussi fidèle que possible des enregistrements, j’ai effectué un double travail de découpage, à partir de la version orale et des notes de cours, c'est-àdire, pour reprendre le vocabulaire barthésien, à partir de la «scription» et de la transcription. Mon travail a consisté à fragmenter les différents textes en unités intelligibles que l'utilisateur devait appeler à partir d'un ou de plusieurs mots-clés ou «concepts» figurant dans une liste. Les deux cents fragments environ correspondaient chacun à une unité de sens et dépendaient des articulations du raisonnement ménagées par Barthes. Tout découpage engage naturellement la responsabilité de l'éditeur, mais c'est toujours l'organisation du cours qui a servi de référence.

La sélection des mots-clés, ou «concepts», que j'ai choisie la plus ouverte possible, m'a amené à jouer sur deux tableaux à la fois, à concilier le savoir du lecteur assidu de Barthes et la culture moins spécialisée du simple curieux. Le premier pensera sans doute aux mots «délicatesse» ou «bathmologie», si récurrents dans l'univers de Barthes. Le second, moins connaisseur, doit pouvoir faire surgir l'information selon des voies moins intentionnelles. Il y aurait, certainement, un très beau travail à faire sur l'usage des index constitués par Barthes dans ses livres. Généralement peu pratiques, ces index si déconcertants cachent autant qu'ils révèlent, combinent les entrées anodines et les portes essentielles. Comme simple commentateur de Barthes, il me fallait opter pour une attitude plus franchement universitaire, ménageant un équilibre périlleux entre la connivence et la naïveté. L'index du site, c'est-à-dire l'ensemble des mots-clés ou «concepts», se devait d'abord d'être pratique.

L'outil informatique permet une grande liberté et une grande diversité d'usages. L'utilisateur pouvait appeler tout mot (nom propre, nom commun...) en "plein texte» et suivre ainsi son propre parcours de recherche. L'ensemble des fragments comportant le mot sélectionné apparaissait à l'écran, comme dans un concordancier. Reproduite en annexe de la notice de présentation du site, une liste des noms propres utilisés par Barthes dans ses notes de cours permettait d'orienter le choix de l'utilisateur. Mais cette liste, seulement incitative ou indicative, ne se confondait pas avec les «concepts», qui relevaient d'un travail éditorial d'une tout autre ambition.

Un index des «concepts» diffère d'un index des notions. En effet, le «concept» n'est pas un simple mot dont le moteur de recherche repérerait les 
occurrences. L'index des concepts proposés sur ce site ne se bornait pas à reproduire l'index des noms propres et des notions figurant dans la version imprimée du cours Comment vivre ensemble. Qu'est-ce donc qu'un «concept $t^{10} »$ ? Donné de préférence au masculin singulier, sans majuscule, le concept, créé par l'éditeur, renvoie tantôt à un mot, tantôt à un ensemble de mots présents dans le texte. La conception de cet index obéissait à une double contrainte : $I^{\circ}$ l'index des concepts ne devait pas se présenter comme un simple index de mots, destiné à guider l'utilisateur dans la constitution de son index personnalisé; $2^{\circ}$ il ne devait pas davantage contraindre le lecteur par une lecture particulière du cours de Barthes.

Choisi selon des critères lexicaux, un concept pouvait d'abord renvoyer à un ensemble de mots formés sur la même racine. Ce type d'expansion, conduit selon des critères lexicaux, n'appelle guère la contestation. Ainsi, le concept «idiorrythmie» renvoyait tantôt au mot «idiorrythmie», tantôt à un ensemble de mots de la même famille («idiorrythmique», «idiorrythme», etc.); le concept «ascétisme» renvoyait également aux mots «ascèse», «ascète», «askèsis»...

Les critères sémantiques relèvent davantage d'un choix éditorial. Ce choix a été guidé par un double souci de pertinence et de discrétion. Un concept simple peut thématiser un ensemble de mots entrant en relation sur le plan sémantique (et non plus lexical). Ainsi, le concept «Bible» renvoyait à tous les mots ou groupes de mots faisant explicitement référence à l'Ancien et au Nouveau Testament: «Adam», «Ève», «Évangiles», «Ceci est mon sang... »; le concept «Orient» renvoyait à toutes les références faites au Proche-Orient (érémitisme chrétien) et à l'Extrême-Orient (bouddhisme de Ceylan, Tao); le concept «Barthes» renvoyait à toutes les allusions que fait Barthes à son œuvre (titres ou citations) ou à sa vie (anecdotes personnelles); le concept «grec» renvoyait à toutes les références au grec ancien (et non à l'ensemble du monde culturel grec); le concept «œuvres» renvoyait exclusivement à la présentation que donne Barthes de son corpus.

Un certain nombre de concepts très généraux ont été sélectionnés en fonction de leur importance : «peinture», «musique», «architecture», «psychanalyse»... Par contre, pour trouver l'ensemble des références au cinéma, art peu représenté dans le cours, il fallait passer par le relais de la liste des noms propres («Fellini»...). Afin de ne pas contraindre le lecteur par des rapprochements trop subjectifs effectués par l'éditeur, seules les références évidentes ont été proposées. Par exemple, le concept «psychanalyse» englobait le champ lexical du mot «psychanalyse», les noms de psychanalystes (Freud, Lacan), le 
vocabulaire propre à la discipline («grand Autre»), mais ne renvoyait pas systématiquement au mot «imaginaire» ou au mot «inconscient» qui ne relèvent que partiellement du domaine psychanalytique.

Les concepts permettaient de rapprocher des passages empruntés à la totalité des séances. Parallèlement à ce dialogue du texte avec lui-même, on a veillé à maintenir la cohérence de chacun des «traits» choisis par Barthes pour structurer son cours. Par exemple, tous les fragments du trait «Domestiques», quel que soit leur contenu, renvoyaient au concept «domestique». L'utilisateur pouvait ainsi bénéficier à la fois du discontinu permis par l'indexation et du continu pensé par l'auteur. En choisissant le concept «domestique», l'utilisateur appelait l'ensemble des fragments constituant le trait «Domestiques» et la totalité des fragments comportant le concept «domestique».

En sélectionnant un concept, l'utilisateur faisait donc apparaître l'ensemble des fragments correspondant à ce choix. Ainsi, le concept «Castorp» permettait de retrouver tous les passages où figure le personnage principal de La Montagne magique, quelle que soit la forme sous laquelle il apparaît («Hans», «Castorp» ou «Hans Castorp»). Une recherche en «plein texte», rappelons-le, laissait à l'utilisateur la liberté de taper chacun des mots que regroupe le concept (par exemple : «Hans»). Concrètement, chaque fragment sélectionné était précédé de toutes les informations permettant de le localiser dans le cours (date, nature du support...) et de la liste de tous les concepts qui lui étaient rattachés. Le choix d'un concept comme «Castorp» donnait lieu à une utilisation simple. Mais des relations plus complexes étaient possibles. Organisés selon un principe d'arborescence, les concepts pouvaient également entrer en relation les uns avec les autres. Par exemple, le concept «Castorp» était systématiquement mis en relation avec d'autres concepts liés à lui sémantiquement. Avec tout fragment correspondant au concept "Castorp», on trouvait indexés les concepts généraux suivants : «La Montagne magique», «Mann» et «roman». Ainsi, en sélectionnant le concept «Castorp», l'utilisateur faisait apparaittre l'ensemble des fragments où figure le personnage; en sélectionnant le concept «La Montagne magique», il faisait apparaitre tous les fragments entrant en relation avec le titre de l'œuvre, le nom d'un personnage («Castorp»), le récit d'un épisode du roman, etc.; en sélectionnant le concept «Mann», il faisait apparaître toutes les références à l'œuvre de Thomas Mann, quel qu'en soit le type de manifestation : titres (La Montagne magique, La Mortà Venise...), nom de l'auteur, noms de personnages ("Castorp»), etc.; en sélectionnant le concept «roman», il faisait apparaitre l'ensemble des fragments qui traitent sous une forme ou sous une autre d'un roman en particulier (La Montagne magique), d'un personnage («Castorp») ou du genre romanesque en général. 
La dernière étape du travail éditorial correspondait à la présentation de quelques informations générales permettant à l'internaute d'entrer plus facilement dans le monde de Barthes (biographie, questionnaire, notice explicative...). Une bibliographie sélective en cinq langues (français, anglais, allemand, italien et espagnol) complétait l'ensemble. Dans la notice biographique, j'avais présenté simultanément la vie et l'œuvre de Barthes pour un public de non-spécialistes, en essayant d'éviter les pièges de la vulgarisation et d'une relation causale réductrice. Le Seuil tenait beaucoup à l'élaboration d'un questionnaire permettant au lecteur d'évaluer ses propres connaissances. Un quiz, un QCM pour Barthes? Le rapprochement parait bien singulier, presque blasphématoire... En excluant le QCM, j'ai fait le choix de questions portant sur le vocabulaire de Barthes, sur ces mots-clés récurrents dans toute l'œuvre dont la définition flottante embarrasse souvent le lecteur. Les définitions proposées n'engageaient bien sûr que l'éditeur : elles appelaient la discussion, le complément, la correction. Il appartenait à chaque lecteur de donner vie à ce site destiné à devenir interactif.

Barthes n'était pas - ou plutôt n'aurait sans doute pas été - un homme de l'ordinateur. Mais le site consacré au Comment vivre ensemble ne lui était pas, je l'espère, infidèle. En effet, ce site accomplissait une des virtualités du cours, offrait à sa manière une nouvelle forme d'idiorrythmie qui s'inscrivait dans le prolongement de l'œuvre. Grâce à la machine, l'utilisateur, à la fois guidé par Barthes et plus modestement par l'éditeur, accomplissait son propre parcours de recherche. Le site proposait le même corpus à tout le monde : à chacun de se l'approprier, à chacun, à la fois libre et orienté, de trouver son propre rythme dans le rythme collectif de la technique informatique et de la pensée barthésienne.

\section{Retour au livre}

Cette liberté du lecteur, la réunion sur un seul support de tous les modes d'expression, sont restées sans lendemain. En septembre 2006, le Seuil a décidé de fermer le site Barthes, mettant fin à une expérience très bien accueillie sur le plan intellectuel, mais ruineuse sur le plan financier. Limité à un seul cours, destiné à un public exclusivement francophone, le site Barthes était payant et son coût élevé limitait l'abonnement aux grandes institutions culturelles (les bibliothèques universitaires, en particulier, qui auraient préféré la solution du CDROM). Il fallait une centaine d'abonnements dans le monde pour rentabiliser l'investissement; après deux ans d'expérience, les résultats n'atteignaient pas la dizaine... On comprend bien dans ces conditions que le Seuil ait préféré mettre 
fin à une entreprise unique dans son genre, mais inadaptée au marché et aux habitudes culturelles. Cet échec commercial pose clairement la question de la viabilité économique de l'Internet littéraire. En démultipliant l'information culturelle, l'outil informatique a incontestablement modifié notre relation matérielle à la diffusion du savoir. Le piratage systématique, la crise des droits d'auteur, la gratuité comme évidence pour tant de jeunes - et moins jeunes internautes, tout cela ne pouvait que compromettre la réussite d'un projet commercial condamné pour survivre à l'équilibre financier...

Partie du manuscrit, transformée en livre, accomplie peut-être par l'outil informatique, la pensée de Barthes, et le surprenant dialogue idiorrythmique qu'elle instaure, tout cet effort pour diffuser un savoir qui ne veut rien «saisir», retourne donc au livre, à l'objet imprimé. Pour prendre connaissance du Comment viure ensemble, il faut désormais revenir à l'édition papier, écouter l'enregistrement des séances, ou consulter les archives de l'IMEC, à Caen... Pour un temps encore, le livre et le disque imposent leur nécessité... 